\title{
CASUÍSTICA DE 10 ANOS DE ANOMALIAS CONGÊNITAS DO SISTEMA NERVOSO EM UM ESTADO BRASILEIRO
}

\section{YEARS CAUSUISTIC OF NERVOUS SYSTEM CONGENITAL ABNOMALIES IN A BRAZILIAN STATE}

\author{
Julio Cesar Campos Ferreira Filho ${ }^{1}$ \\ Baruc Silveira Veras Macedo ${ }^{2}$ \\ Sávio Sales Silva Silveira ${ }^{3}$ \\ Caio Victor Queiroga Barreto ${ }^{4}$ \\ Fred Brasileiro Costa Júnior \\ Vanessa Erika Abrantes Coutinho ${ }^{6}$
}

RESUMO: Objetivo: Este trabalho se propõe a reunir dados dos casos notificados de Espinha Bífida (EB) e Outras Malformações Congênitas do Sistema Nervoso (OMF), em 10 anos na Paraíba, além de analisar seus fatores predisponentes e sua gravidade ao nascimento. Método: Trata-se de um estudo transversal ecológico que analisa uma década (2008-2017) de casos notificados no Sistema Único de Saúde (SUS) de EB e OMF no estado da Paraíba, Brasil. Os dados coletados no sistema DATASUS e analisados descritivamente e inferencialmente $(p<0,05)$ usando $o$ software SPSS 20.0. Resultados: Não houve associação entre $E B(N=98)$ e as variáveis estudadas $(p>0,05)$, entretanto houve uma evolução nos valores de Apgar $1^{\circ}-5^{\circ}$ minuto $(p<0,01)$. OMF $(N=586)$, esteve relacionada a etnia/raça $(p=0,012)$, duração da gestação $(p=0,044)$, piores indicadores Apgar ( $1^{\circ}$ minuto: $\rho=-0,118 ; 5^{\circ}$ minuto: $\rho=-0,193 ; p<0,01)$ e houve evolução do índice $8-10 \mathrm{em} 15,7 \%$ do $1^{\circ}$ minuto para o $5^{\circ}$ minuto de vida $(p<0,01)$. Adicionalmente identificou-se aumento do Apgar com o passar dos anos avaliados $(p<0,05)$ e maiores indicadores Apgar $\left(1^{\circ}\right.$ minuto: $\rho=0,692$; $5^{\circ}$ minuto: $\rho=0,493 ; p<0,01$ ) com relação a melhor assistência pré-natal. Conclusão: No período de 2008-2017, na Paraíba-Brasil, foram registrados 684 casos de Espinha bífida e outras malformações congênitas do sistema nervoso. Outras malformações estiveram mais presentes em pardos e proporcionou piores valores de Apgar $1^{\circ}-5^{\circ}$ minutos. Houve uma tendência de melhora nos valores de

\footnotetext{
${ }^{1}$ Acadêmico de Medicina da Faculdade Santa Maria - FSM, Cajazeiras, Brasil.

${ }^{2}$ Acadêmico de Medicina da Faculdade Santa Maria - FSM, Cajazeiras, Brasil.

${ }^{3}$ Acadêmico de Medicina da Faculdade Santa Maria - FSM, Cajazeiras, Brasil.

${ }^{4}$ Acadêmico de Medicina da Faculdade Santa Maria - FSM, Cajazeiras, Brasil.

${ }^{5}$ Acadêmico de Medicina da Faculdade Santa Maria - FSM, Cajazeiras, Brasil.

${ }^{6}$ Docente do curso de Medicina da Faculdade Santa Maria - FSM, Cajazeiras, Brasil.
} 
Apgar com o passar dos anos avaliados e com uma melhor assistência pré-natal nestas crianças com malformações congênitas do sistema nervoso.

Palavras chave: Anormalidades Congênitas. Sistema Nervoso. Epidemiologia. Saúde Pública.

ABSTRACT: Objective: This article aims to gather data from notified cases in 10 years, Paraíba - Brazil, of Spina Bifida (SB) and Other Congenital Malformations of the Nervous System (OCM) and to analyze of its predisposing factors and severity at birth. Method: This is a cross-sectional and ecological study that analyzes a decade (2008-2017), of cases notified in the Unified Health System of Brazil (known as SUS) by DATASUS (database of Brazilian SUS), of Spina Bifida (SB) and Other Congenital Malformations of the Nervous System (OCM) in the state of Paraiba, Brazil. The data were evaluated descriptively and inferentially $(p<0.05)$ using the SPSS 20.0 software. Results: There was no association between $S B(N=98)$ and the variables studied ( $p>0.05)$, however there was an increase in the Apgar values from $1^{\circ}-5^{\circ}$ minute $(p<0.01)$. OCM $(N=586)$, was related to Ethnicity $(p=0.012)$, duration of pregnancy $(p=0.044)$, worst Apgar values (1st minute: $\rho=-0.118$; 5 th minute: $\rho=-$ $0.193 ; p<0$, 01) and evolution of the $8-10$ index in $15.7 \%$ from the 1 st minute to the 5th minute of life $(p<0.01)$. Additionally, an increase in the Apgar score was obtained over the years observed ( $p<0.05)$ and higher Apgar values (1st minute: $\rho=0.692$; 5th minute: $\rho=0.493 ; p<0.01)$ in relation to more complete prenatal care. Conclusion: In the period of 2008-2017, in Paraíba, 98 cases of Spina bifida and 586 cases of other congenital malformations of the nervous system were registered. Other malformations were more present in females, browns and provided worse Apgar scores from 1st to 5th minutes of birth. There was a tendency of improvement in Apgar values over the years evaluated and with better prenatal care in these children with congenital malformations of the nervous system.

Keywords: Congenital Abnormalities. Nervous System. /epidemiology. Public Health. 


\section{INTRODUÇÃO}

Malformações ou anomalias congênitas (AC) são definidas como distúrbios essencialmente estruturais, funcionais, metabólicos ou comportamentais durante 0 período do desenvolvimento embrionário que geralmente necessitam de intervenções estéticas, reabilitadoras ou cirúrgicas de forma significativa, mas que diante de distúrbios brandos podem ter grau de necessidade da intervenção reduzido ou eletivo (SADLER, LANGMAN, 2016).

Estes distúrbios de desenvolvimento têm caráter ambiental, genético ou multifatorial. As causas genéticas abrangem 3 a cada 10 casos de AC, destacandose irregularidades cromossômicas e mutações gênicas. Drogas, fármacos doenças infecciosas, poluentes ambientais, pesticidas e doenças maternas durante a gravidez constituem $15 \%$ dos casos e mais da metade é ocasionada por condição multifatorial incluindo interações entre agentes teratogênicos e alterações genéticas. Além destes fatores, a idade materna (abaixo de 20 anos e acima de 35 anos) durante a gestação é fator predisponente, elevando o risco e severidade das anomalias congênitas (ANDRADE et al., 2017; MARINHO et al., 2016; RAMOS, OLIVEIRA, CARDOSO, 2008; RODRIGUES et al., 2014; SADLER, LANGMAN, 2016).

Estima-se uma prevalência mundial de distúrbios de desenvolvimento em torno de 3 a $5 \%$ do número de nascidos vivos, e destes, 1-2\% expressam defeitos graves à saúde (NUNES, 2010). No Brasil, encontrou-se uma prevalência variável entre 1 e $2,5 \%$ a depender da região estudada (ALMEIDA et al., 2016; JUNIOR et al., 2017) retratando um quadro de grande importância em saúde e dependente da variabilidade de fatores ambientais que devem ser melhor elucidados.

Entre as ACs o sistema nervoso é o mais acometido no Brasil (REIS et al., 2015; WESTPHAL et al., 2016) e se configura como principal causa de óbito na infância (ALMEIDA et al., 2016). Em nível local, há estudos que abordam os casos de malformações congênitas em paraibanos (LIMA et al., 2019; LIMA, VIANNA, 
MORAES et al., 2019), no entanto, abordam um período máximo de 6 anos com um aspecto mais relacionado à regionalidade do que à investigação/explicação dos agentes causais. Portanto, diante da importância em saúde destas anomalias congênitas, pesquisas que abranjam uma grande faixa temporal e investiguem relações causais e suas tendências para facilitar o processo de tomada de decisão para conseguinte prevenção, são de grande relevância. Deste modo, este trabalho se propõe a explorar no período de 10 anos os casos de malformações congênitas do sistema nervoso na Paraíba e analisar possíveis fatores predisponentes ao acometimento de anormalidades congênitas do sistema nervoso.

\section{MÉTODOS}

Trata-se de um estudo transversal ecológico que descreve e analisa, no período de 10 anos (2008-2017), casos notificados de Espinha Bífida (EB) e Outras malformações congênitas associadas ao sistema nervoso (OMF) que foram compatíveis com a vida no estado da Paraíba-PB, Brasil. Os dados foram acessados no Sistema de Informações sobre nascidos vivos (SINASC) do DATASUS.

Os dados desta pesquisa são de caráter secundários, com coleta realizada no banco de dados online do Sistema de Informações em Saúde - SIS no período de 1 a 31 de outubro de 2019. Foram escolhidas as seguintes variáveis: sexo do recémnato (RN), Malformação ("Espinha bífida" e "Outras malformações do sistema nervoso"), idade da mãe no momento do parto (faixas etárias entre 10-44 anos), etnia/raça do recém-nascido (referida pelos pais), nível de assistência pré-natal (inexistente, intermediária, adequada), tempo total da gestação (em trimestres) e Índice Apgar no primeiro e quinto minuto ao nascer.

As informações coletadas foram analisadas descritivamente e inferencialmente por meio dos testes: Shapiro-Wilk, Komolgorov-Smirnov, KruskalWallis, Wilcoxon, $X^{2}$ (qui-quadrado de Pearson) e Correlação de Spearman. Para tanto, foi utilizado o software Statistical Package for Social Sciences (SPSS ${ }^{\circledR}$ ) versão 
25.0. Os resultados foram interpretados considerando um intervalo de confiança de 95\%, e nível de significância de 5\% (p-valor<0,05).

\section{RESULTADOS}

Ao longo de 10 anos (2008-2017), na Paraíba-Brasil, foram registrados 98 casos de EB distribuídos de forma regular e com leve aumento na maioria dos anos. Ocorreram 586 casos de OMF, ressaltando o acentuado valor nos anos 2015 $(n=153 ; 26,11 \%)$ e $2016(n=115 ; 19,62 \%)$ (Quadro 1). Considerando a EB, houve uma maior prevalência em recém-natos $(R N)$ do sexo feminino ( $n=57 ; 58,2 \%)$, referidos pelos pais como pardos $(n=79 ; 84,0 \%)$ e com mães entre 20-24 anos $(n=29 ; 29,6 \%)$. Apenas 64,8\% (n=34) das mães fizeram o pré-natal adequadamente e todos os RN ( $n=98)$ nasceram durante o $3^{\circ}$ trimestre de gravidez. No entanto, ao longo da década avaliada, não houve associação da EB com as variáveis estudadas $(p>0,05)$. Houve uma evolução $(p<0,01)$ no índice Apgar ao $1^{\circ}$ minuto de vida do RN (valor $8-10 \mathrm{com} n=60 ; 62,5 \%$ ) para o $5^{\circ}$ minuto (valor $8-10 \mathrm{com} n=89 ; 92,7 \%$ ) (Tabela 1).

Quanto ao registro conjunto de OMF houve associação com a Etnia/raça referida pela mãe $(p=0,012)$, duração da gestação $(p=0,044)$ e os dois indicadores Apgar $(p<0,01)$, que na primeira avaliação, 3 a cada $10 \mathrm{RN}$ com malformação do sistema nervoso apresentaram escore igual ou inferior à 5. Destaca-se também que, na duração da gestação, $9 \mathrm{RN}$ nasceram durante o $2^{\circ}$ trimestre e 7 durante após 0 $3^{\circ}$ Trimestre. Assim como na EB, houve uma evolução $(p<0,01)$ do RN em torno de $15 \%$ do $1^{\circ}$ minuto (índice $8-10: n=298 ; 51,6 \%$ ) para o $5^{\circ}$ minuto de vida (índice $8-10$ : $\mathrm{n}=387 ; 67,3 \%$ ) (Tabela 1).

Ao comparar EB com OMF não houve diferença na distribuição entre as variáveis sexo $(p=0,297)$, etnia $(p=0,169)$, idade da mãe ao parto $(p=0,770)$, nível de assistência pré-natal $(p=0,457)$, e duração de gestação $(p=0,254)$. No entanto, houve diferença nos indicadores Apgar $1^{\circ}$ minuto $(p=0,002)$ e $5^{\circ}$ minuto $(p<0,001)$. 
Por fim, recém-nascidos com OMF apresentaram pior quadro geral de saúde e vitalidade ao expressarem menores escores nos Índices Apgar $1^{\circ}$ minuto $(\rho=-$ $0,118 ; p=0,02)$ e $5^{\circ}$ minuto $(\rho=-0,193 ; p<0,01)$; uma forte propensão de melhora no indicador Apgar após 5 minutos de vida $(\rho=0,801, p<0,01)$; um leve aumento dos valores Apgar com o passar dos anos dos dados coletados $(p<0,05)$; e moderada correlação entre melhor assistência pré-natal e maiores indicadores Apgar $1^{\circ}$ minuto $(\rho=0,692 ; p<0,01)$ e Apgar $5^{\circ}$ minuto $(\rho=0,493 ; p<0,01)$ (Tabela 2).

\section{DISCUSSÃO}

A maior parte das mulheres avaliadas tiveram filhos entre 20-24 anos, e este perfil assemelha-se ao encontrado no estado do Rio Grande do Norte (LIMA et al., 2018). Assim como em trabalhos anteriores, houve uma maior prevalência de crianças do sexo feminino com AC (LIMA, VIANA, MORAES, 2019, LIMA et al., 2019) e houve um aumento considerável no número de casos de malformações congênitas do sistema nervoso no período entre 2014-2016 (LIMA, VIANNA, MORAES, 2019; LIMA et al., 2019). Adicionalmente, a melhora irregular nos escores dos Índices Apgar $1^{\circ}$ e $5^{\circ}$ minuto nos recém-nascidos com alguma AC no período de 2010-2016 é corroborada na literatura (LIMA, VIANNA, MORAES, 2019). Entretanto, houve incompatibilidade de comparação entre os resultados, principalmente ao considerar a relação entre as variáveis, devido à diferença na categorização e análises realizadas entre os estudos.

Esta elevação inesperada no número de casos pode ter ocorrido em razão do surto de exposição intrauterina ao vírus Zika que esteve relacionado à elevação no coeficiente de prevalência de nascidos vivos com microcefalia na região Nordeste, principalmente na Paraíba, a partir de 2015 (MARINHO et al., 2016). Contudo, a construção de conhecimento sobre esta relação teratogênica do vírus Zika, aliada à tomada de decisão preventiva governamental durante o processo da gravidez, são hipóteses à considerável redução, em 2017, e retorno ao número de casos de $A C$ ao padrão pré-surto viral. 
O atual estudo, por utilizar um banco de dados secundários apresenta limitações relacionadas ao processo de subnotificação ou erros de registro na base de dados em saúde, DATASUS (CARVALHO, SIQUEIRA, 2019; LIMA et al., 2018). Isto pode explicar uma maior concentração de casos em cidades de grande porte, onde além do número populacional, apresentam uma maior rigidez e solidez dos sistemas de vigilância epidemiológicas em saúde. Todavia, ressalta-se que o SINASC é uma base de dados oficial de nível nacional com preenchimento obrigatório, que permite a exploração, comparação dos resultados e tem passado por processos de capacitação na coleta e minimização de problemas quanto a notificação.

Estudos brasileiros têm exposto grandes índices de mortalidade (14 a 32\%) para os fetos com algum tipo de AC severa, como por exemplo as que acometem o sistema nervoso central ou malformações múltiplas (BARROS et al., 2012; VIEIRA et al., 2012). Aliada a elevada mortalidade, merece atenção a necessidade de intervenção especializada por vezes durante toda vida do paciente, dada cronicidade do distúrbio de desenvolvimento e grande impacto psicológico, social e econômico para o indivíduo e família afetados (SADLER, LANGMAN, 2016; SANTOS et al., 2011). Nesta perspectiva, a identificação e abordagem preventiva diante de fatores de risco ambientais para o surgimento de AC são alternativas que devem ser valorizadas, tendo em vista a maior acessibilidade e que defeitos genéticos são passíveis de detecção durante o pré-natal (AMORIM et al., 2006).

Embora não tenha sido encontrada relação entre o pré-natal e AC do sistema nervoso $(p<0,05)$, o número máximo de apenas $65 \%$ das mães com assistência prénatal adequada é um dado alarmante que pode ocasionar problemas para os recémnascidos. Segundo a Organização Mundial da Saúde (OMS), uma assistência adequada está pautada em uma quantidade igual ou superior a seis consultas prénatal e isto é necessário para o processo de avaliação precoce de riscos de antecedentes de mortalidade perinatal, malformações congênitas, risco de prematuridade ou morte uterina. (OMS, 2013; RODRIGUES et al., 2014). Deste modo, isto pode explicar as correlações encontradas entre melhores assistências pré-natal com a menor tendência de nascimentos prematuros, e melhores indicadores Apgar em 1 e 5 minutos $(p<0,05)$. 
O índice Apgar é aferido no primeiro e quinto minuto de vida do recém-nato e representa um rápido e válido método de predição das condições gerais primárias e secundárias de saúde neonatal. Escores menores que 3 são indicativos de grande risco de paralisia cerebral e morte neonatal (MOSTER et al., 2001). Portanto, compõe a rotina da avaliação de saúde após o nascimento. Nesta perspectiva, considerando os resultados, houve evolução nos valores entre $1^{\circ}$ e $5^{\circ}$ minuto $(p<0,01)$ e uma forte correlação de maiores escores no $1^{\circ}$ minuto com $5^{\circ}$ minuto. Isto representa uma evolução no quadro geral de saúde da amostra de recém-nascidos com AC do sistema nervoso.

Adicionalmente, baixos resultados de Apgar $1^{\circ}$ minuto podem representar condições vitais por depressão temporária do recém-nato e, deste modo, o $5^{\circ}$ minuto é mais eficaz como preditor de sobrevivência do bebê (MOSTER et al., 2001). Portanto, os menores escores encontrados para Apgar 1 minuto em RN com AC de sistema nervoso podem expressar uma imaturidade funcional deste sistema de caráter temporário no que se refere às condições vitais básicas. Entretanto, vale salientar que o conjunto de malformações do sistema nervoso apresentou maior gravidade clínica por expressar menores valores de Apgar, quando comparado com espinha bífida, o que pode ser comprovado pela severidade das malformações do sistema nervoso, como por exemplo, microcefalia que comprovadamente manifesta baixas pontuações Apgar (GROSS et al., 1978; MARINHO et al., 2016).

No período avaliado, aproximadamente 2 a cada 10 mães tinham menos de 20 anos e 1 a cada 10 têm mais de 35 anos durante a gestação de crianças com AC do sistema nervoso (espinha bífida e demais). Ou seja, 30\% das mães do estudo estavam em uma faixa etária de risco para prematuridade, morte neonatal e $A C$ (ANDRADE et al., 2017; MARINHO et al., 2016; RAMOS, OLIVEIRA, CARDOSO, 2008; RODRIGUES et al., 2014; SADLER, LANGMAN, 2016) especialmente por anormalidades cromossômicas. Contudo, embora a gravidez precoce esteja relatada como predisponente para problemáticas neonatais, foi encontrada uma tendência de redução das contagens de Apgar $1^{\circ}$ minuto, quanto maior a idade da mãe durante a gestação, maior a probabilidade de um bebê com AC de sistema nervoso.

Portanto, o seguinte estudo permitiu a construção de uma visão retrospectiva dos casos notificados de anomalias congênitas do sistema nervoso nos últimos 10 
anos de um estado brasileiro (Paraíba), fornecendo uma leitura epidemiológica sobre possíveis fatores de risco para o surgimento e severidade de malformações do sistema nervoso. Deste modo, com a leitura dos resultados deste trabalho é possível elaborar medidas de saúde com enfoque preventivo e consequentemente melhorar a qualidade de vida dos futuros recém-nascidos da região. Vale salientar que, por se tratar de condições gerais, há possibilidade de extensão das relações causais deste estudo para tomadas de decisão preventivas em outros centros urbanos, no entanto os resultados devem ser lidos com cautela, pois dados locais são mais efetivos por atuar diretamente nos pontos críticos para cada região abordada.

Nesta perspectiva, no período de 2008-2017, na Paraíba-Brasil, foram registrados 98 casos de Espinha bífida e 586 casos de outras malformações congênitas do sistema nervoso. A maioria dos casos foi de recém-natos do sexo feminino, pardos e com mães entre 20-24 anos. Houve uma tendência de evolução dos escores Apgar $1^{\circ}$ para $5^{\circ}$ minuto e maior severidade clínica para o conjunto de outras anomalias do sistema nervoso que esteve associada com etnia e duração da gestação.

Deste modo, este estudo vem ressaltar o quadro geral da distribuição de Espinha Bífida e Malformações envolvendo o Sistema Nervoso em nascidos vivos na Paraíba em uma década e a investigação de relações que permitam identificar potenciais grupos de risco (por exemplo: mães muito jovens no momento do parto, incompleta assistência pré-natal, nascimento prematuro ou gestação acima de 9 meses). Portanto, este estudo permite identificar estes grupos, ressaltar a problemática local e incentivar a condução de medidas de saúde necessárias para prevenção e acompanhamento das crianças com estas afecções embriológicas, para maior expectativa e qualidade de vida dos afetados. 


\section{REFERÊNCIAS BIBLIOGRÁFICAS}

1. ALMEIDA, L.F.G; ARAÚJO, E.J.R.; CROTT, G.C.; OKIDO, M.M.; BEREZOWSKI, A.T.; et al. Epidemiological risk factors and perinatal outcomes of congenital anomalies. Revista Brasileira de Ginecologia e Obstetrícia, v. 38, n. 7, p. 348-355, 2016.

2. AMORIM, M.M.R.; VILELA, P.C.; SANTOS, A.R.V.D.; LIMA, A.L.M.V.; MELO, E.F.P.; et al. Impacto das malformações congênitas na mortalidade perinatal e neonatal em uma maternidade-escola do Recife. Revista Brasileira de Saúde Materno Infantil, v. 6, n. Supl 1, p. S19-S25, 2006.

3. ANDRADE, A.M.; RAMALHO, A.A.; OPITZ, S.P.; MARTINS, F.A.; KOIFMAN, R.J. Anomalias congênitas em nascidos vivos. Revista Brasileira em Promoção da Saúde, v. 30 , n. 1, p. 1-11, 2017.

4. BARROS, M.L.; FERNANDES, D.A.; MELO, E.V.; PORTO, R.L.S.; MAIA, M.C.A.; et al. Malformações do sistema nervoso central e malformações associadas diagnosticadas pela ultrassonografia obstétrica. Radiologia Brasileira, v. 45, n. 6, p. 309-314, 2012.

5. CARVALHO, R.R.S.S.; SIQUEIRA, J.H. Caracterização epidemiológica da esquistossomose no estado do Espírito Santo de 2010 a 2015. Brazilian Journal of Health Research, v. 21, n. 1, p. 95-103, 2019.

6. GROSS, S.J.; KOSMETATOS, N.; GRIMES, C.T.; WILLIAMS, M.L. Newborn head size and neurological status. Predictors of growth and development of low birth weight infants. A.M.A. American journal of diseases of children, v. 132, n. 8, p. 753-756, 1978.

7. JUNIOR, E.P.P.; LUZ, L.A.; GUIMARÃES, M.A.P.; TAVARES, L.T.; BRITO, T.R.S; SOUZA GF. Prevalência e fatores associados às anomalias congênitas em recém-nascidos. Revista brasileira em promoção da saúde, v. 30, n. 3, p. 1-9, 2017.

8. LIMA, L.M.M.; MELO, A.C.O.; VIANNA, R.P.T.; MORAES, R.M. Análise espacial das anomalias congênitas do sistema nervoso. Cadernos Saúde Coletiva, v. 7, n. 3, p. 257-263, 2019.

9. $\quad$ LIMA, L.M.M.; VIANNA, R.P.T.; MORAES, R.M. Análise espacial e espaço-temporal das anomalias congênitas do sistema nervoso no Estado da Paraíba entre os anos de 2010 a 2016. Journal of Human Growth and Development, v. 29, n. 2, p. 169-176, 2019.

10. LIMA, N.A.; SILVA, C.F.; SANTOS, M.M.; FERNANDES, T.A.A.M.; LUCENA, E.E.S. Perfil Epidemiológico das Malformações Congênitas em Recém-Nascidos no Estado do Rio Grande do Norte no Período de 2004 a 2011. Revista Brasileira de Ciências da Saúde, v. 22, n. 1, p. 45-50, 2018.

11. MARINHO, F.; ARAÚJO, V.E.M.; PORTO, D.L.; FERREIRA, H.L.; COELHO, M.R.S.; et al. Microcefalia no Brasil: prevalência e caracterização dos casos a partir do Sistema de Informações sobre Nascidos Vivos (Sinasc), 2000-2015. Epidemiologia e Serviços de Saúde, v. 25, n. 4 , p. $701-712,2016$.

12. NUNES, M.D. Perfil epidemiológico das malformações congênitas em recémnascidos no estado do Tocantins no período de 2004 a 2008. Dissertação (Dissertação em Ciências da Saúde) - Universidade de Brasília. Brasília, p. 110. 2010.

13. RAMOS, A.P.; OLIVEIRA, M.N.D.; CARDOSO, J.P. Prevalência de malformações 
congênitas em recém-nascidos em hospital da rede pública. Revista Saúde.Com, v. 4, n. 1, p. 27-42, 2008.

14. REIS, L.V.; ARAÚJO JÚNIOR, E.; GUAZZELLI, C.A.F.; CERNACH, M.C.S.P.; TORLONI, M.R.; MORON, A.F. Anomalias congénitas identificadas ao nascimento em recém-nascidos de mulheres adolescentes. Acta Médica Portuguesa, v. 28, n. 6, p. 708-714, 2015.

15. RODRIGUES, L.S.; LIMA, R.H.S.; COSTA, L.C.; BATISTA, R.F.L. Características das crianças nascidas com malformações congênitas no município de São Luís, Maranhão, 20022011. Epidemiologia e Serviços de Saúde, v. 23, n. 2, p. 295-304, 2014.

16. SADLER, Thomas W.; LANGMAN, Jan. Embriologia médica 13. ed. Rio de Janeiro: Guanabara Koogan, 2016.

17. SANTOS, S.R.; DIAS, I.M.A.V.; SALIMENA, A.M.O.; BARA, V.M.F. A vivência dos pais de uma criança com mal formações congênitas. Revista Mineira de Enfermagem, v. 15, n. 4, p. 491-497, 2011.

18. VIEIRA, M.S.M.; SIEBERT, E.C.; CEGLIO, W.Q.G.W.; ALMEIDA, M.H.; BATISTA, T.S.; FREITAS, P.F. Dificuldades para a identificação da causa do óbito fetal: como resolver? Revista Brasileira de Ginecologia e Obstetrícia, v. 34, n. 9, p. 403-408, 2012.

19. WESTPHAL, F.; FUSTINONI, S.M.; PINTO, V.L.; MELO, P.S.; ABRAHÃO, A.R. Associação da idade gestacional com a opção pela interrupção da gravidez de fetos com anomalias incompatíveis com a sobrevida neonatal. Einstein, v. 14, n. 3, p. 311-316, 2016. 


\section{ANEXOS}

Tabela 1: Análise descritiva e inferencial dos casos notificados de Espinha Bífida e Outras malformações do Sistema Nervoso $(p<0,05)$.

\begin{tabular}{|c|c|c|c|c|c|c|c|}
\hline & & $\begin{array}{c}\text { Espinha } \\
\text { Bífida (\%) }\end{array}$ & p-valor & $\begin{array}{l}\text { Total } \\
(\%)\end{array}$ & OMF & p-valor & $\begin{array}{c}\text { Total } \\
(\%)\end{array}$ \\
\hline \multirow{2}{*}{ Sexo } & Masculino & $41(41,8)$ & \multirow{2}{*}{0,549} & \multirow{2}{*}{$\begin{array}{c}98 \\
(100)\end{array}$} & $277(47,5)$ & \multirow{2}{*}{0,099} & \multirow{2}{*}{$\begin{array}{l}583 \\
(100)\end{array}$} \\
\hline & Feminino & $57(58,2)$ & & & $306(52,5)$ & & \\
\hline \multirow{4}{*}{ Etnia } & Branco & $15(16,0)$ & \multirow{4}{*}{0,059} & \multirow{4}{*}{$\begin{array}{c}94 \\
(100)\end{array}$} & $61(10,7)$ & \multirow{4}{*}{$0,012^{*}$} & \multirow{4}{*}{$\begin{array}{c}572 \\
(100)\end{array}$} \\
\hline & Pardo & $79(84,0)$ & & & $500(87,4)$ & & \\
\hline & Negro & $0(0,0)$ & & & $9(1,6)$ & & \\
\hline & Indígena & $0(0,0)$ & & & $2(0,3)$ & & \\
\hline \multirow{4}{*}{$\begin{array}{l}\text { Nível de } \\
\text { assistência } \\
\text { Pré-natal } \\
\text { (NAPN) }\end{array}$} & Inexistente & $0(0,0)$ & \multirow{4}{*}{0,182} & \multirow{4}{*}{$\begin{array}{c}54 \\
(100)\end{array}$} & $3(0,9)$ & \multirow{4}{*}{0,274} & \multirow{4}{*}{$\begin{array}{c}327 \\
(100)\end{array}$} \\
\hline & Inadequada & $11(20,4)$ & & & $82(25,1)$ & & \\
\hline & Intermediária & $8(14,8)$ & & & $29(8,9)$ & & \\
\hline & Adequada & $35(64,8)$ & & & $213(65,1)$ & & \\
\hline \multirow{3}{*}{$\begin{array}{l}\text { Duração da } \\
\text { gestação }\end{array}$} & $2^{\circ}$ trimestre & $0(0,0)$ & \multirow{3}{*}{1,0} & \multirow{3}{*}{$\begin{array}{c}98 \\
(100)\end{array}$} & $9(1,5)$ & \multirow{3}{*}{$0,044^{*}$} & \multirow{3}{*}{$\begin{array}{l}586 \\
(100)\end{array}$} \\
\hline & $3^{\circ}$ trimestre & $98(100)$ & & & $570(97,3)$ & & \\
\hline & $\begin{array}{ll}\text { Após } & 3^{\circ} \\
\text { trimestre }\end{array}$ & $0(0,0)$ & & & $7(1,2)$ & & \\
\hline \multirow{7}{*}{$\begin{array}{lr}\text { Idade da } \\
\text { mãe ao } \\
\text { parto (anos) }\end{array}$} & $10-14$ & $2(2,0)$ & \multirow{7}{*}{0,737} & \multirow{7}{*}{$\begin{array}{c}98 \\
(100)\end{array}$} & $1(0,2)$ & \multirow{7}{*}{0,174} & \multirow{7}{*}{$\begin{array}{c}585 \\
(100)\end{array}$} \\
\hline & $15-19$ & $17(17,3)$ & & & $121(20,6)$ & & \\
\hline & $20-24$ & $29(29,6)$ & & & $167(28,5)$ & & \\
\hline & $25-29$ & $19(19,4)$ & & & $135(23,0)$ & & \\
\hline & $30-34$ & $22(22,4)$ & & & $101(17,2)$ & & \\
\hline & $35-39$ & $7(7,1)$ & & & $50(8,5)$ & & \\
\hline & $40-44$ & $2(2,0)$ & & & $11(1,9)$ & & \\
\hline \multirow{4}{*}{$\begin{array}{l}\text { Apgar } \\
\text { minuto }\end{array}$} & $0-2$ & $3(3,1)$ & \multirow{4}{*}{0,078} & \multirow{4}{*}{$\begin{array}{c}96 \\
(100)\end{array}$} & $91(15,8)$ & \multirow{4}{*}{$<0,01^{*}$} & \multirow{4}{*}{$\begin{array}{c}577 \\
(100)\end{array}$} \\
\hline & $3-5$ & $7(7,3)$ & & & $82(14,2)$ & & \\
\hline & 6-7 & $26(27,1)$ & & & $106(18,4)$ & & \\
\hline & $8-10$ & $60(62,5)$ & & & $298(51,6)$ & & \\
\hline & $0-2$ & $2(2,1)$ & & & $60(10,4)$ & & \\
\hline Apgar & $3-5$ & $2(2,1)$ & 0575 & 96 & $55(9,6)$ & $0001 *$ & 575 \\
\hline minuto & 6-7 & $3(3,1)$ & 0,015 & $(100)$ & $73(12,7)$ & $<0,01$ & (100) \\
\hline & $8-10$ & $89(92,7)$ & & & $387(67,3)$ & & \\
\hline $\begin{array}{l}\text { Apgar } 1^{\circ} \mathrm{min} \\
\text { p-valor }\end{array}$ & Apgar $5^{\circ} \mathrm{min}$ & & $<0,01^{*}$ & & & $<0,01^{*}$ & \\
\hline
\end{tabular}

Legenda: EB - Espinha Bífida

OMF - Outras malformações do Sistema Nervoso. 
Tabela 2: Correlações das variáveis estudadas entre si $(p<0,05)$.

\begin{tabular}{|c|c|c|c|c|c|c|c|c|c|}
\hline & & Sexo & Etnia & NAPN & $\begin{array}{c}\text { Tempo } \\
\text { gestação }\end{array}$ & $\begin{array}{l}\text { Idade } \\
\text { Mãe }\end{array}$ & $\begin{array}{c}\text { Apgar } \\
1 \mathrm{~min}\end{array}$ & $\begin{array}{l}\text { Apgar } \\
5 \mathrm{~min}\end{array}$ & Ano \\
\hline \multirow[t]{2}{*}{$A C$} & $\begin{array}{l}\rho- \\
\text { Spearma } \\
\mathrm{n}\end{array}$ &,- 040 & ,069 &,- 014 &,- 008 &,- 011 &,$- 118^{* *}$ &,$- 193^{* *}$ & ,002 \\
\hline & $\mathrm{p}$-valor & ,298 & ,077 & ,790 & ,836 & ,770 & ,002 & , 000 &, 963 \\
\hline \multirow[t]{2}{*}{ NAPN } & $\begin{array}{l}\rho- \\
\text { Spearma } \\
\mathrm{n}\end{array}$ & & & & ,211 &,- 043 &, $692^{* *}$ &, $493^{* *}$ &,- 004 \\
\hline & $\mathrm{p}$-valor & & & & ,000 & ,398 & , 000 &, 000 & ,937 \\
\hline \multirow[t]{2}{*}{$\begin{array}{l}\text { Tempo } \\
\text { gestação }\end{array}$} & $\begin{array}{l}\rho- \\
\text { Spearma } \\
\mathrm{n}\end{array}$ & & & & &,- 014 &,- 017 &,- 012 & ,064 \\
\hline & p-valor & & & & & ,707 & ,663 & ,760 & ,096 \\
\hline \multirow[t]{2}{*}{$\begin{array}{l}\text { Idade da } \\
\text { mãe }\end{array}$} & $\begin{array}{l}\text { - } \\
\text { Spearma } \\
\mathrm{n}\end{array}$ & & & & & &,$- 081^{*}$ &,- 032 & ,007 \\
\hline & $\mathrm{p}$-valor & & & & & & ,035 & ,411 & 860 \\
\hline \multirow[t]{2}{*}{$\begin{array}{l}\text { Apgar } 1^{\circ} \\
\text { min }\end{array}$} & $\begin{array}{l}\rho- \\
\text { Spearma } \\
\mathrm{n}\end{array}$ & & & & & & &, $807^{\star *}$ &, $104^{* *}$ \\
\hline & $\mathrm{p}$-valor & & & & & & & ,000 & 007 \\
\hline \multirow[t]{2}{*}{$\begin{array}{l}\text { Apgar } 5^{\circ} \\
\text { min }\end{array}$} & $\begin{array}{l}\rho- \\
\text { Spearma } \\
n\end{array}$ & & & & & & & &, $076^{*}$ \\
\hline & $\mathrm{p}$-valor & & & & & & & & ,049 \\
\hline
\end{tabular}

Legenda: AC - Anomalia congênita do sistema nervoso; NAPN - Nível de assistência Pré-natal. 


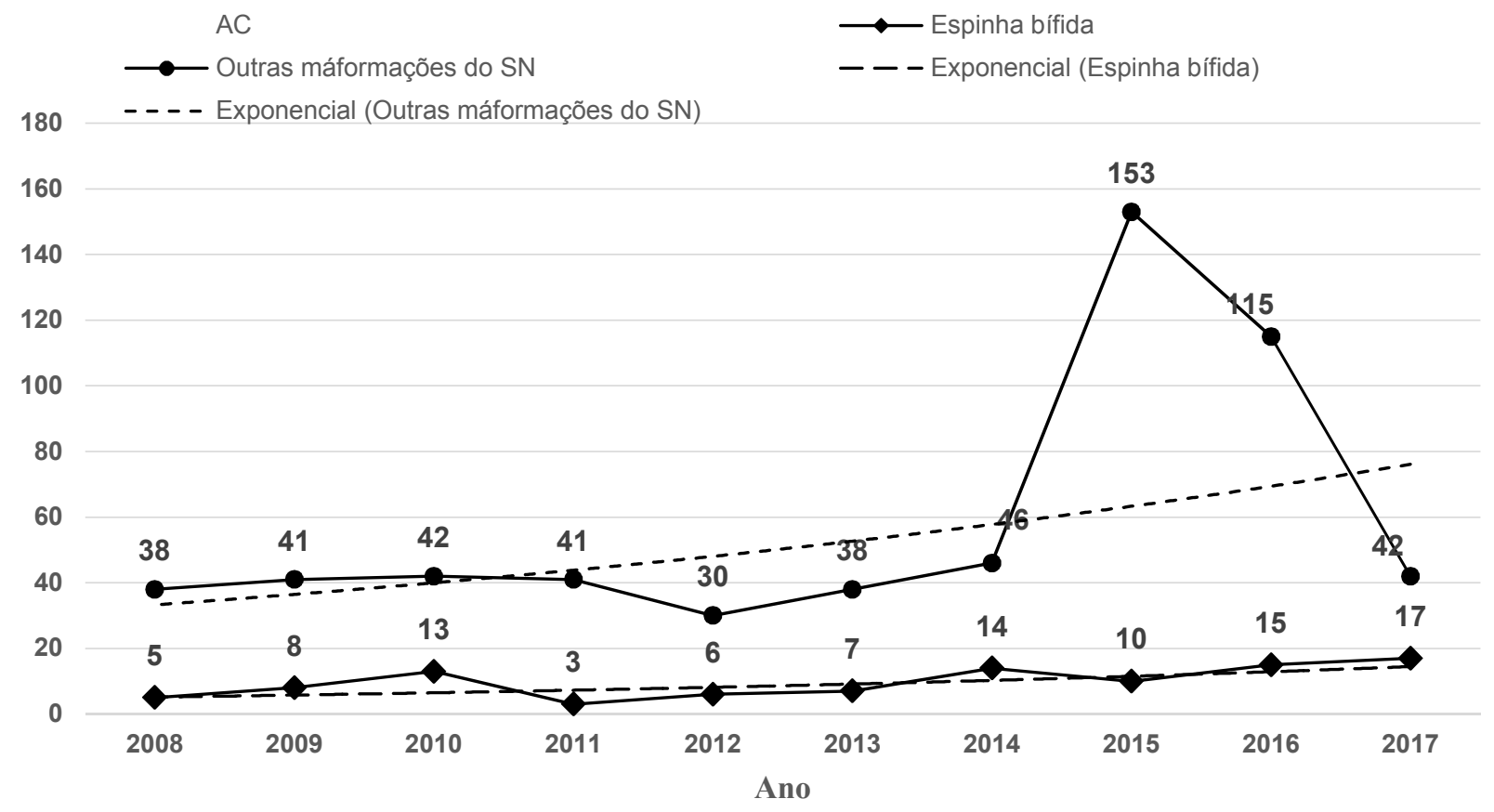

Legenda: AC - Anomalia congênita do Sistema Nervoso.

Quadro 1: Distribuição dos casos de Espinha bífida e Outras malformações. 